\title{
Utilização de diferentes critérios para avaliação do potencial de biodisponibilidade de metais pesados em sedimentos de fundo do alto Tietê (São Paulo)
}

\author{
Graziela Meneghel de Moraes ${ }^{1}$, Jefferson Mortatti ${ }^{2}$ \& Renato Alessandro Lopes ${ }^{1}$
}

\begin{abstract}
Resumo Diferentes critérios para avaliação da biodisponibilidade de metais pesados nos sedimentos de fundo da bacia do alto rio Tietê foram utilizados a partir do método de sulfetos volatilizados após ataque ácido a frio (AVS) e extração simultânea de metais pesados (SEM). Dentre os critérios utilizados, o SEM-AVS, normalizado pela matéria orgânica, mostrou-se mais adequado para determinação de biodisponibilidade de metais pesados evidenciando uma não biodisponibilidade nos pontos de amostragem de Ponte Nova, Biritiba, Mogi das Cruzes e Pirapora. Entretanto, os valores-guia de qualidade de sedimento (TEL e PEL) quando aplicados, apresentaram efeito de toxicidade ao longo da bacia. Isso ocorre devido ao fato de que os valoresguia não levaram em consideração o fundo geoquímico natural local, provando que os critérios TEL e PEL superestimam a biodisponibilidade de metais pesados em sedimentos de fundo.
\end{abstract}

Palavras-chave: Biodisponibilidade, Critérios, Metais Pesados, Rio Tietê, Sedimentos.

\begin{abstract}
Use of different criteria for evaluation of potential bioavailability of heavy metals in bottom sediments of upper Tietê (São Paulo). Different criteria to evaluate bioavailability of heavy metals in sediments of Upper Tietê River Basin were used from acid volatile sulfide (AVS) and simultaneously extracted metals (SEM) methods. Among the criteria, SEM-AVS, normalized by organic matter, was more appropriate for bioavailability determination of heavy metals showing a non-bioavailability at Ponte Nova, Biritiba, Mogi das Cruzes and Pirapora sampling points. However, the sediment quality guidelines (TEL and PEL) showed toxicity effects along the basin, due to the guideline values do not take into account the local natural background level, demonstrating that TEL and PEL criteria overestimate the bioavailability of heavy metals in bottom sediments.
\end{abstract}

Keywords: Bioavailability, Criteria, Heavy metals, Tietê River, Sediments.

INTRODUÇÃO Os sedimentos são considerados importantes compartimentos de geoacumulação de metais ou fonte de liberação de metais para um sistema aquático (Murray et al. 1999). Por ter capacidade de retenção, os sedimentos podem ser indicadores de qualidade de água e registrar os efeitos de emissões antrópicas (Oliveira et al. 2006), pois desempenham papel fundamental na dinâmica do transporte físico, acumulação e biodisponibilização de contaminantes (Almeida \& Rocha 2006). A presença desses contaminantes nos sedimentos de corpos hídricos representa um potencial latente da degradação continuada desses ambientes, mesmo que a coluna d'água e sua biota não apresentem concentrações desses elementos, acima daquelas previstas na legislação vigente da qualidade da água (Mozeto 2001).

A retenção dos metais ocorre pela incorporação destes aos sedimentos de fundo por processos de adsorção e complexação, principalmente em sedimentos de granulometria fina ou com teor elevado de matéria orgânica. Esta incorporação ocorre pelo fato de que a matéria orgânica presente nos sedimentos normalmente é oxidada por bactérias sulfato redutoras, que usam os sulfatos como receptores de elétrons. Essa redução leva à formação de sulfeto de hidrogênio $\left(\mathrm{H}_{2} \mathrm{~S}\right)$ e monosulfetos secundários (Lacerda \& Marins 2006).

De acordo com Di Toro et al. (1990) e Allen et al. (1993), em condições anóxidas, os óxidos de ferro são reduzidos a formas iônicas ferrosas, que reagem com o sulfeto de hidrogênio, formando uma grande variedade de minerais de sulfetos de ferro, incluindo a pirita $\left(\mathrm{FeS}_{2}\right)$ e a forma amorfa $(\mathrm{FeS})$, sendo esta a mais comum, nos sedimentos de fundo aquático.

Com a dissociação do $\mathrm{FeS}$, na fase aquosa, alguns cátions metálicos divalentes $(\mathrm{Cu}, \mathrm{Co}, \mathrm{Cr}, \mathrm{Zn}, \mathrm{Cd}$, $\mathrm{Ni}$ e $\mathrm{Pb}$ ), por apresentarem potencial de oxirredução maior, reagem com o sulfeto formando sulfetos mais insolúveis que os de ferro. Essas ligações controlam as concentrações de metais pesados e a biodisponibilidade dos mesmos na fase sortiva sedimento-água.

Outro método adotado, para avaliação de toxicidade de metais pesados em sedimentos de fundo fluvial, é o proposto pela Companhia de Tecnologia e Saneamento Ambiental (CETESB), onde as concentrações totais de metais pesados são comparadas

1 - Programa de Pós-Graduação Química na Agricultura e no Ambiente, Centro de Energia Nuclear na Agricultura, Universidade de São

Paulo, Piracicaba (SP), Brasil. E-mail: gmmoraes@cena.usp.br, ralopes@cena.usp.br

2 - Centro de Energia Nuclear na Agricultura, Universidade de São Paulo, Piracicaba (SP), Brasil. E-mail: jmortatti@cena.usp.br 
diretamente com valores-guia de qualidade de sedimentos (VGQS), propostos pelo Conselho Canadense de Ministérios do Meio Ambiente (CCME), gerados a partir de um banco de dados (químicos e toxicológicos) disponíveis na América do Norte (Cunha \& Calijuri 2008). Os valores limites foram derivados a partir de bancos de dados de efeitos e não efeitos, onde foram definidas três faixas de concentração química, aquelas que são raramente, ocasionalmente e frequentemente associadas com efeitos biológicos diversos. Os dois valores-guia do CCME são: TEL (Threshold Effect Le$v e l$ ), nível de efeito limiar abaixo do qual não ocorre efeito adverso à comunidade biológica e os contaminantes associados ao sedimento, que estiverem dentro desta faixa de concentração, não são considerados de efeito significativo para os organismos aquáticos, sendo calculado como a média geométrica da fração inferior a $15 \%$ da concentração dos dados de efeito e $50 \%$ dos dados de não efeito; e, PEL (Probable Effect Level), nível de efeito provável à ocorrência de efeitos adversos, representando o limite inferior da faixa de concentração de poluentes químicos que usualmente, ou sempre, estão associados com efeitos adversos à biota, calculados a partir da média geométrica de 50\% da concentração dos dados de efeito e $85 \%$ dos dados de não efeito (CCME 1995).

O presente estudo teve por objetivo a avaliação do potencial de biodisponibilidade dos principais metais pesados $\mathrm{Cu}, \mathrm{Co}, \mathrm{Cr}, \mathrm{Zn}, \mathrm{Cd}, \mathrm{Ni}$ e $\mathrm{Pb}$ nos sedimentos de fundo do alto rio Tietê, em perfil de profundidade utilizando diferentes critérios, envolvendo os metais pesados extraídos simultaneamente (SEM) com os sulfetos volatilizados (AVS) após ataque ácido a frio e também a extração total de metais. Desta forma, foi possível estimar o potencial de biodisponibilidade em perfil de profundidade, utilizando diferentes critérios de qualidade de sedimentos, nos pontos de amostragem do reservatório de Ponte Nova, Biritiba, Mogi das Cruzes e reservatório de Pirapora, ao longo da bacia de drenagem do alto rio Tietê.

ÁREA DE ESTUDO O rio Tietê é o maior e mais importante do Estado de São Paulo, principalmente por seu potencial hidroelétrico e de navegabilidade, devido ao fato de suas nascentes, apesar de estarem a $22 \mathrm{~km}$ do oceano Atlântico, atravessa todo o estado de São Paulo, de leste a oeste, até desaguar no rio Paraná, na divisa do Mato Grosso do Sul.

A área de estudo compreendeu a bacia de drenagem do Alto rio Tietê (UGRHI-6), com uma área de $5.985 \mathrm{~km}^{2}$, posicionada de acordo com as coordenadas geográficas de $23^{\circ} 14^{\prime}$ a $24^{\circ}$ de latitude sul e $45^{\circ} 50^{\prime}$ a $47^{\circ} 01^{\prime}$ de longitude oeste, cujas nascentes do rio Tietê estão localizadas próximas ao município de Salesópolis, a $840 \mathrm{~m}$ de altitude até o reservatório de Pirapora, possuindo $250 \mathrm{~km}$ de extensão (Fig. 1).

Conforme pode ser observado, quatro estações de amostragens foram definidas: reservatório de Ponte Nova $\left(23^{\circ} 35^{\prime} 33,3^{\prime \prime}\right.$ S e $45^{\circ} 58^{\prime} 3$ ”'W), Biritiba $\left(23^{\circ} 33^{\prime} 52^{\prime \prime} \mathrm{S}\right.$ e $\left.46^{\circ} 00^{\prime} 59^{\prime \prime} \mathrm{W}\right)$, Mogi das Cruzes $\left(23^{\circ} 30^{\prime} 50^{\prime \prime} \mathrm{S}\right.$ e $\left.46^{\circ} 12^{\prime} 12^{\prime \prime} \mathrm{W}\right)$ e reservatório de Pirapora $\left(23^{\circ} 23^{\prime} 43^{\prime \prime} \mathrm{S}\right.$ e $\left.47^{\circ} 00^{\prime} 17^{\prime \prime} \mathrm{W}\right)$, tais pontos foram

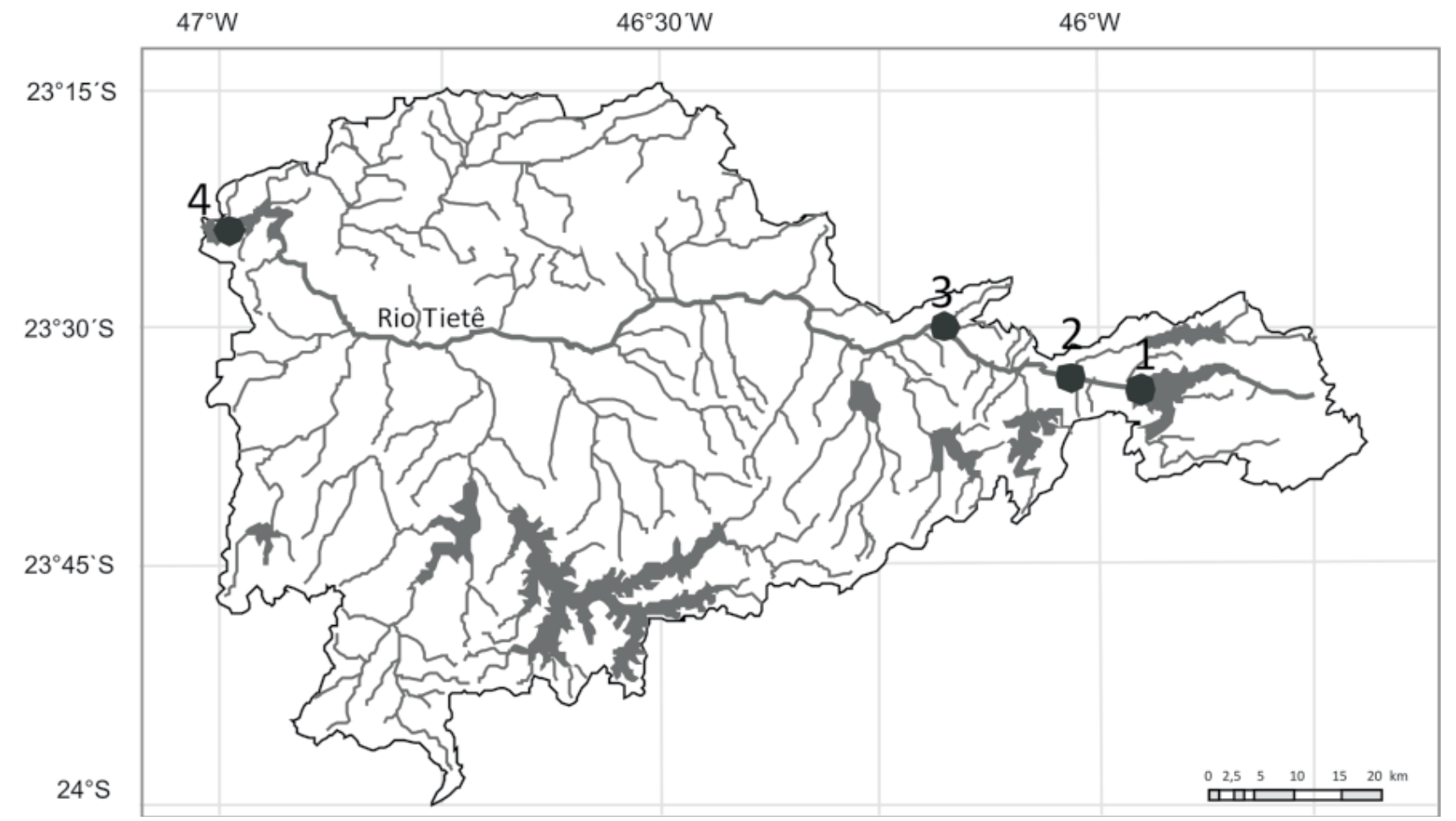

Figura 1 - Localização das estações de amostragem na bacia do Alto rio Tietê: 1 - Reservatório de Ponte Nova, 2 - Biritiba, 3 - Mogi das Cruzes e 4 - Reservatório de Pirapora. 
escolhidos a fim de se ter uma relação de causa e efeito com as principais fontes de contribuição antrópica.

A região do alto rio Tietê possui 36 municípios com destaque para a região metropolitana de São Paulo, que possui aproximadamente 27 milhões de habitantes (mais de $10 \%$ da população do país), onde estima-se que $37,7 \mathrm{~m}^{3} / \mathrm{s}$ de esgoto público e particular são lançados na bacia sem tratamento prévio (DAEE 2006). É possível observar que não foram determinados pontos de amostragem entre 3 e 4 , pois é a região onde estão sendo feitas dragagem dos sedimentos, devido ao projeto de diminuição das enchentes.

A bacia de drenagem do Alto rio Tietê encontrase assentada no Planalto Atlântico, onde se destacam as rochas dos embasamentos cristalinos representadas basicamente por granitos, gnaisses e quartzitos. Os principais tipos de solos são representados pelo argissolo vermelho-amarelo, álico, textura médio-argilosa; latossolo vermelho-amarelo, álico textura argilosa e junto às nascentes cambissolos álicos, organossolos e gleissolos (EMBRAPA 1999). A região apresenta a montante, junto ao reservatório de Ponte Nova, alguns fragmentos do maciço florestal Atlântico ainda preservados. Segundo a classificação de Köpper, o clima predominante é o Cwa com o predomínio da estiagem no inverno e de chuvas no verão.

PROTOCOLO DE AMOSTRAGEM E ANÁLITICO As amostragens dos sedimentos de fundo na bacia do alto Tietê foram realizadas em triplicata nas estações previamente definidas (estações 1, 2, 3 e 4), utilizando um amostrador tipo torpedo. O que permitiu amostragens em perfis de profundidade, o período das amostragens corresponde a abril de 2008, sendo as coletas realizadas no canal central e mais profundo do rio, para cada ponto de amostragem. As amostras obtidas foram armazenadas nos tubos de PVCs, que se encontram dentro do amostrador, lacradas e mantidas a $4^{\circ} \mathrm{C}$. No laboratório, as amostras foram compostas a cada centímetro para uma melhor representatividade do perfil amostrado.

O método utilizado para a determinação de sulfetos volatilizados, após ataque ácido a frio (AVS) e extração simultânea de metais (SEM), seguiu os procedimentos descritos por Di Toro et al. (1992) e Allen et al. (1993), após modificações propostas por Mortatti et al. (2008), as quais incluem as determinações de AVS por condutividade elétrica. Para as análises foram utilizados $1 \mathrm{~g}$ do sedimento úmido para cada amostra de sedimento analisado ao longo do perfil amostrado.

Após a determinação do AVS as soluções residuais foram filtradas à vácuo, em filtros milipore de acetato-celulose $(0,45 \mu \mathrm{m})$ e conduzidos para a análise dos metais pesados $\mathrm{Cu}, \mathrm{Co}, \mathrm{Cr}, \mathrm{Zn}, \mathrm{Cd}, \mathrm{Ni}$ e $\mathrm{Pb}$ por espectrometria de emissão atômica com plasma acoplado indutivamente (ICP OES), sendo possível determinar a soma de metais pesados extraídos (SEM).

Também foi utilizada a extração total de metais pesados proposta por Samuel et al. (1985), em perfil de profundidade, para a qual foi utilizado $100 \mathrm{mg}$ de amostra de sedimento calcinada a $1.000^{\circ} \mathrm{C}$ por $30 \mathrm{~min}$ em cadinhos de platina com os fundentes tretaborato de lítio (400 mg) e metaborato de lítio (200 mg). A mistura foi homogeneizada e colocada numa mufla à $1.000^{\circ} \mathrm{C}$ novamente por $30 \mathrm{~min}$. Após o resfriamento, o fundido é solubilizado com a adição de $25 \mathrm{ml}$ de ácido clorídrico (1M) e completado o volume de $50 \mathrm{ml} \mathrm{com}$ água deionizada. A determinação dos metais pesados totais foram determinados por espectrometria de emissão atômica com plasma acoplado indutivamente (ICP OES). Para controle da qualidade analítica e do processo de extração foram utilizados as concentrações do branco analítico e o material de referência internacional Soil-7 (IAEA), sendo obtidos os seguintes porcentuais médios de recuperação para extração e análise em triplicata: $\mathrm{Cr}$ $(92,3 \pm 5,1 \%), \mathrm{Cu}(94,1 \pm 3,2 \%), \mathrm{Co}(95,3 \pm 6,4 \%), \mathrm{Ni}$ $(97,4 \pm 3,4 \%), \mathrm{Zn}(103 \pm 9,2 \%)$ e $\mathrm{Pb}(107,1 \pm 4,8 \%)$.

\section{DESENVOLVIMENTO METODOLÓGICO Di-} ferentes critérios para a avaliação do potencial de biodisponibilidade de metais pesados em sedimentos fluviais podem ser utilizados envolvendo principalmente os metais extraídos simultaneamente (SEM), com os sulfetos volatilizados (AVS) após ataque ácido a frio.

A agência de Proteção Ambiental dos Estados Unidos (EPA) adota a Teoria do Equilíbrio de Partição, para determinação de biodisponibilidade, estabelecendo o critério de qualidade de sedimentos levando em consideração o equilíbrio estabelecido entre a partição de metais entre os sedimentos e os sulfetos volatilizáveis por acidificação (AVS) (USEPA 2000).

A razão SEM/AVS, descrita por Di Toro et al. (1992), foi inicialmente utilizada considerando que os valores da razão SEM/AVS $>1$ indicam um potencial de biodisponibilidade, enquanto SEM/AVS $<1$ indica que existem sulfetos suficientes para total complexação dos metais pesados existentes. Posteriormente, de acordo com o trabalho de Hansen et. al. (1996), passou a ser utilizada a diferença SEM-AVS onde foi considerado o valor 1,7 como valor guia de qualidade de sedimento. Considerando que SEM-AVS $>1,7$ indica um potencial de biodisponibilidade e SEM-AVS $<1,7$ indica uma não biodisponibilidade, diferentemente do primeiro método, esta formulação tem a vantagem de verificar a capacidade de ligação dos metais com o AVS mesmo quando as concentrações de SEM e AVS forem baixas.

Atualmente, foi proposto pela USEPA (2000) e Di Toro et al. (2005) o método onde a diferença SEMAVS é normalizada pelo carbono orgânico total, que influencia na partição de metais existentes no sedimento, promovendo uma correção na estimativa da biodisponibilidade de metais pesados em sedimentos de fundo, sendo que (SEM-AVS)/C $\mathrm{C}_{\text {org. }}>130$ significa que não existem sulfetos suficientes para total complexação, ou seja, indica potencial de biodisponibilidade, enquanto $(\mathrm{SEM}-\mathrm{AVS}) / \mathrm{C}_{\mathrm{org}}<130$ indica que não há potencial de biodisponibilidade.

Atualmente, não existem valores-guia de qualidade de sedimentos de fundo no Brasil, porém, a CETESB, 
Companhia de Tecnologia e Saneamento Ambiental do Estado de São Paulo, adota os valores propostos pelo Conselho Canadense de Ministérios do Meio Ambiente (CCME) que elaborou um protocolo de derivação e princípios canadenses para a qualidade dos sedimentos e para proteção da vida aquática (CCME 1995). Tal protocolo estabelece dois valores-guia, para concentração total de metais pesados: TEL (Threshold Effect Level), ou seja, nível de efeito limiar, abaixo deste não ocorre efeito adverso à comunidade biológica; e PEL (Probable Effect Level), nível de efeito provável à ocorrência de efeitos adversos para os organismos. Na faixa entre TEL e PEL situam-se os valores onde, ocasionalmente, espera-se a ocorrência de tais efeitos (Silvério et al. 2006, Mozeto et al. 2007).

A tabela 1 apresenta os valores de referência de qualidade propostos para comparação com as concentrações totais dos metais pesados extraídos (TEL e PEL).

$\mathrm{O}$ presente estudo procurou comparar os diferentes métodos descritos anteriormente, utilizando os diferentes valores-guia propostos.

RESULTADOS E DISCUSSÃO Na figura 2 podem ser observadas as distribuições de SEM e AVS, em perfis de profundidade para a estação de amostragem de Ponte Nova, na bacia do alto rio Tietê, de acordo com os três critérios de avaliação propostos.

Foi possível verificar que a utilização da razão SEM/AVS se mostrou superestimada com relação ao equilíbrio proposto (SEM/AVS $=1)$, ao longo de todo perfil amostrado, apresentando um valor médio de 4,82 $\pm 1,68 \mu \mathrm{mol} . \mathrm{g}^{-1}$ configurando um elevado potencial de biodisponibilidade de metais pesados. Tal ocorrência se mostrou influenciada pelas baixas concentrações dos metais extraídos e, principalmente, do enxofre volatilizado.

O critério de avaliação SEM-AVS se mostrou mais adequado e não influenciado pelas baixas concentrações observadas, apresentando um valor médio de $0,40 \pm 0,14 \mu \mathrm{mol} \cdot \mathrm{g}^{-1}$ ao longo do perfil amostrado, sendo todos os valores verificados inferiores ao índice limite proposto (SEM-AVS $=1,7$ ), mostrando, portanto, a não biodisponibilidade de metais. Da mesma forma, o critério de avaliação (SEM-AVS)/C $\mathrm{C}_{\text {org }}$, que normaliza os resultados pelo teor de carbono orgânico, se mostrou adequado ao estudo proposto, caracterizando a não

Tabela 1 - Valores-guia de referência (TEL e PEL) para sedimentos de fundo.

\begin{tabular}{lccccccc}
\hline $\begin{array}{l}\text { Valores de } \\
\text { Referência }\end{array}$ & $\mathrm{Cu}$ & $\mathrm{Co}$ & $\mathrm{Cr}$ & $\mathrm{Zn}$ & $\mathrm{Cd}$ & $\mathrm{Ni}$ & $\mathrm{Pb}$ \\
\cline { 2 - 7 } & \multicolumn{7}{c}{$\left(\mu \mathrm{g} \cdot \mathrm{g}^{-1}\right)$} \\
\hline TEL & 35,7 & 10,0 & 37,3 & 123,1 & 0,6 & 18,0 & 35,0 \\
\hline PEL & 197,0 & & 90,0 & 315,0 & 3,5 & 35,9 & 91,3 \\
\hline
\end{tabular}

Smith et al. (1996) biodisponibilidade de metais pesados ao longo do perfil estudado, com um valor médio de $11,07 \pm 3,70 \mu \mathrm{mol}^{-\mathrm{g}^{-1}}$, muito inferior ao índice limite de $130 \mu \mathrm{mol} . \mathrm{g}^{-1}$.

As distribuições de AVS e SEM, em perfil de profundidade, para a estação de amostragem de Biritiba, pode ser observado na figura 3 , de acordo com os três critérios de avaliação proposto.

Para a estação de amostragem de Biritiba, foi possível verificar que a utilização da razão SEM/AVS mostrou valores superiores a 1 , sendo que, ao longo do perfil, a média obtida foi de 1,68 $\pm 0,89 \mu \mathrm{mol} \cdot \mathrm{g}^{-1}$, configurando um elevado potencial de biodisponibilidade de metais pesados devido às baixas concentrações desses metais e do enxofre volatilizado, assim como observado anteriormente para a estação de amostragem de Ponte Nova. O critério de avaliação de SEM-AVS apresentou valor médio de $0,07 \pm 0,09 \mu \mathrm{mol} . \mathrm{g}^{-1}$, sendo inferior ao índice limite de 1,7 , mostrando, portanto, uma não biodisponibilidade. O mesmo ocorreu quando da normalização da matéria orgânica, apresentando um valor médio de 2,16 $\pm 4,44 \mu \mathrm{mol} . \mathrm{g}^{-1}$, sendo muito inferior ao índice limite de não biodisponibilidade de $130 \mu \mathrm{mol} . \mathrm{g}^{-1}$.

A figura 4 apresenta as distribuições de SEM e AVS nos perfis de profundidade para a estação de amostragem de Mogi das Cruzes, no alto da bacia do rio Tietê, de acordo com os três critérios de avaliação propostos.

$\mathrm{Na}$ estação de amostragem de Mogi das Cruzes, área onde ocorre a presença de contribuição antrópica, em termos de efluentes domésticos despejados sem tratamentos nos cursos d'água, apresentou um valor médio de $1,76 \pm 3,55 \mu \mathrm{mol} . \mathrm{g}^{-1}$, sendo possível verificar que a razão $\mathrm{SEM} / \mathrm{AVS}$ se mostrou abaixo do índice limite de 1 até os $16 \mathrm{~cm}$ de profundidade, principalmente devido aos elevados teores de AVS. Entretanto, a partir dessa profundidade, os valores da razão se mostraram superiores ao índice limite sendo influenciados pelas baixas concentrações de AVS, muito próximas ao limite de determinação, mesmo apresentando baixos teores de SEM observados.

Novamente, o critério de avaliação SEM-AVS mostrou todos os valores observados ao longo do perfil, inferiores ao índice limite de 1,7, sendo que ao longo do perfil, a média foi de $-2,13 \pm 3,18 \mu \mathrm{mol} . \mathrm{g}^{-1}$, mostrando uma não biodisponibilidade de metais pesados. $\mathrm{O}$ critério de avaliação (SEM-AVS)/C $\mathrm{C}_{\text {org }}$ também mostrou uma não biodisponibilidade de metais pesados, apresentando um valor médio de $-119,35 \pm 238,26 \mu$ mol. $\mathrm{g}^{-1}$ ao longo do perfil amostrado, sendo inferior ao índice limite de $130 \mu \mathrm{mol} . \mathrm{g}^{-1}$.

$\mathrm{Na}$ figura 5 podem ser observadas as distribuições de SEM e AVS nos perfis de profundidade, referentes à estação de amostragem de Pirapora, na bacia do alto rio Tietê, de acordo com os três critérios de avaliação propostos.

Foi possível verificar, para a estação de amostragem de Pirapora (a jusante da contribuição antrópica da grande São Paulo), que os critérios utilizados para a avaliação da biodisponibilidade de metais pesados apresentaram resultados similares, com ocorrência de possível toxicidade nos sedimentos mais profundos 


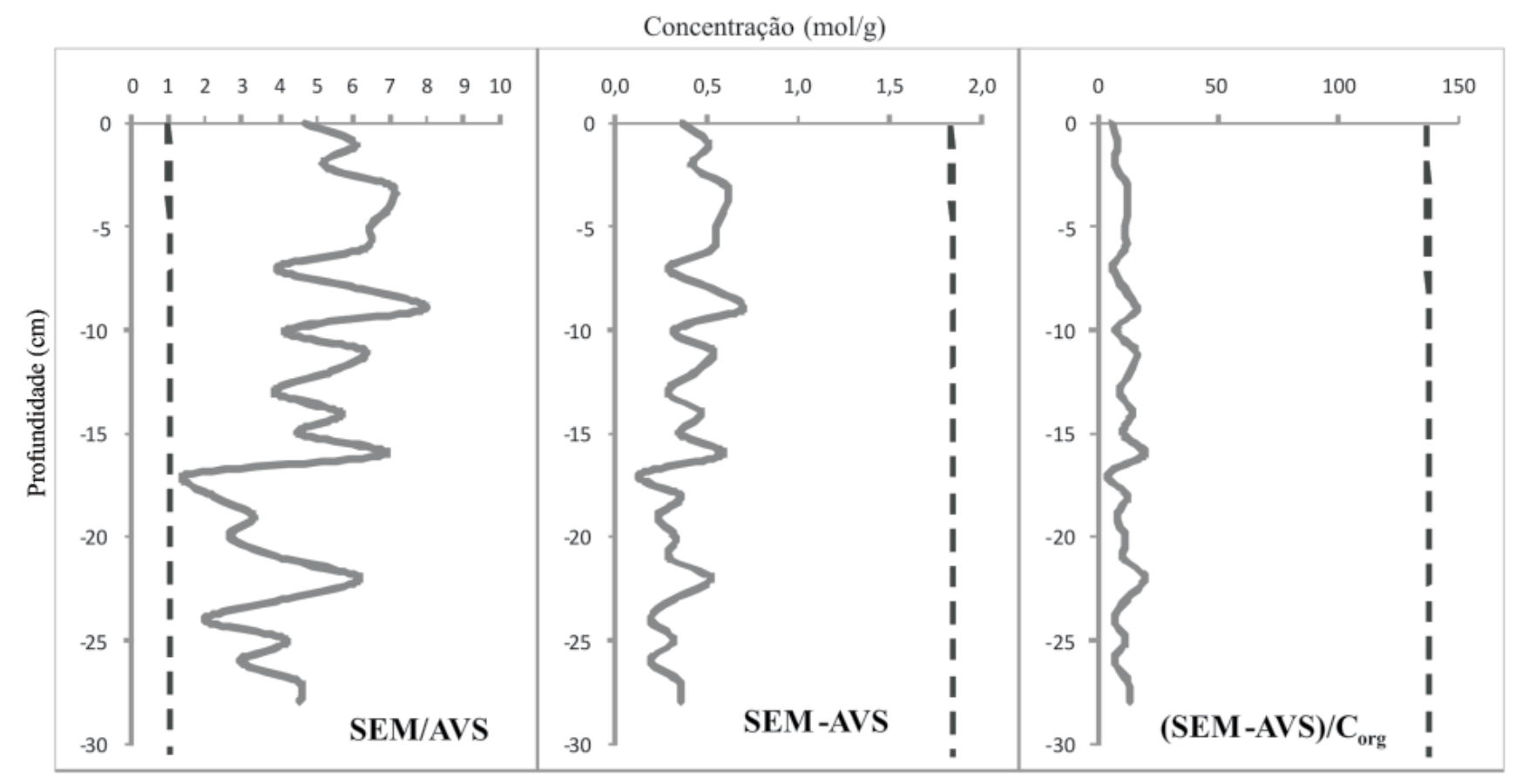

Figura 2 - Relacionamento SEM e AVS, determinados nos sedimentos de fundo, na estação de amostragem de Ponte Nova, em perfil de profundidade, utilizando os critérios de avaliação propostos.

(maior que $15 \mathrm{~cm}$ ), embora o critério que utiliza a normalização pela matéria orgânica deva ser empregado.

Mesmo com elevadas concentrações de $\mathrm{Cu}$, $\mathrm{Co}, \mathrm{Cr}, \mathrm{Zn}, \mathrm{Cd}$, $\mathrm{Ni}$ e $\mathrm{Pb}$, observadas ao longo do perfil amostrado em Pirapora, as respectivas concentrações de AVS se mostraram suficientes para a complexação desses metais até $16 \mathrm{~cm}$ de profundidade. Concentrações elevadas de AVS também foram verificadas por Bevilacqua et al. 2009, Silva et al. 2002 e Silvério 2003, na estação de Pirapora, próximo à represa de Rasgão.

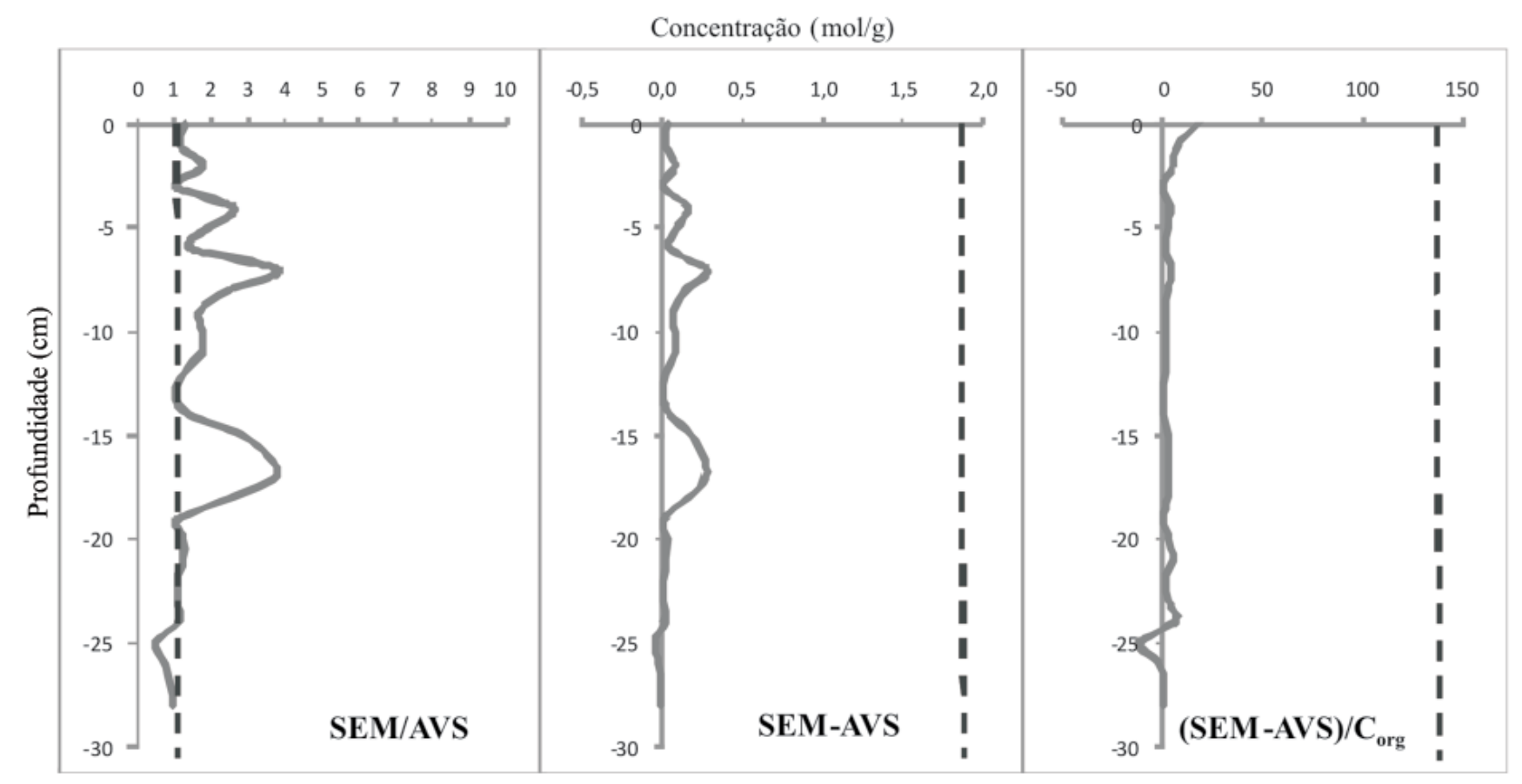

Figura 3 - Relacionamento SEM e AVS, determinados nos sedimentos de fundo, na estação de amostragem de Biritiba, em perfil de profundidade, utilizando os critérios de avaliação propostos. 


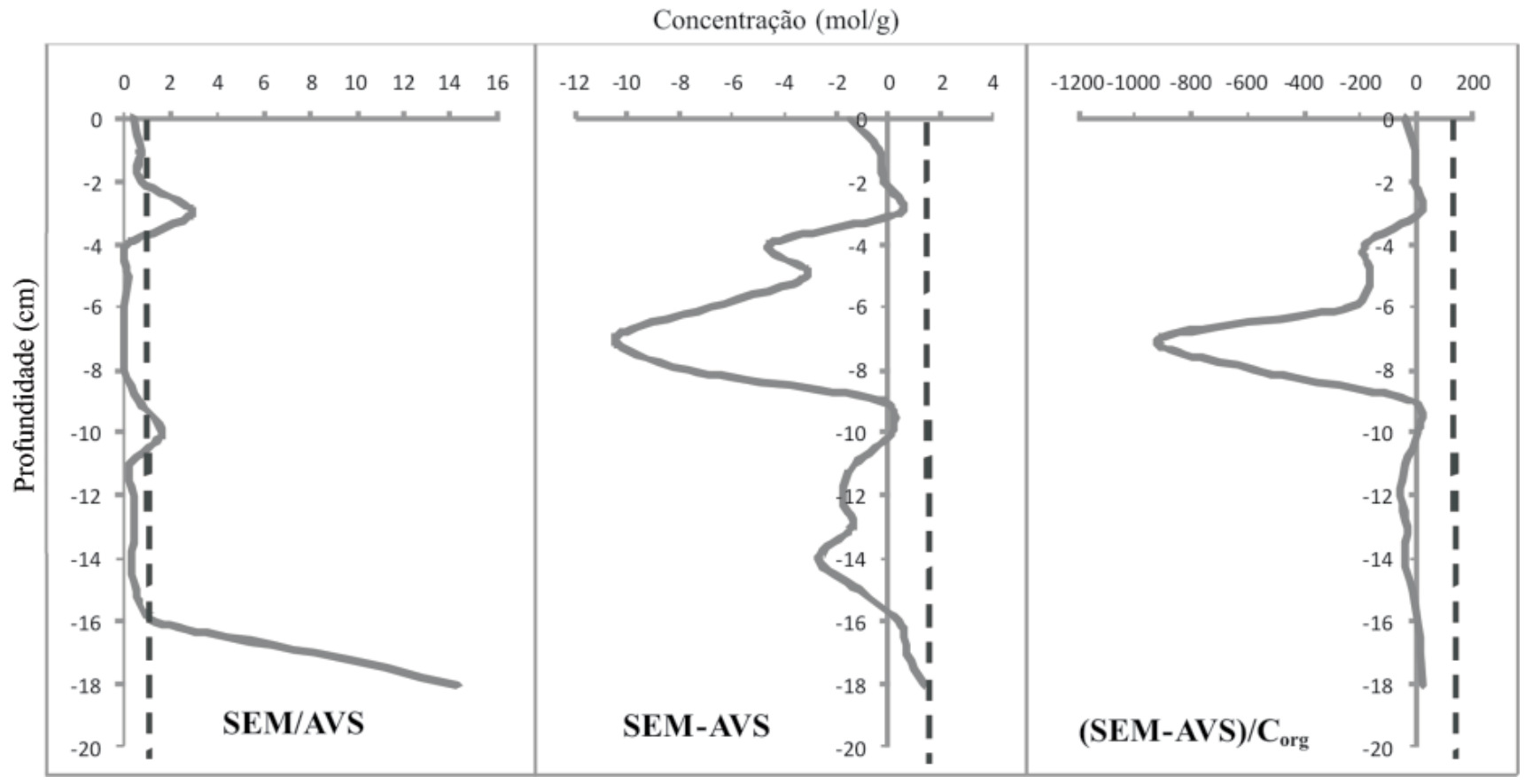

Figura 4 - Relacionamento SEM e AVS, determinados nos sedimentos de fundo, na estação de amostragem de Mogi das Cruzes, em perfil de profundidade, utilizando os critérios de avaliação propostos.

Paradoxalmente parece que, neste caso, a alta concentração de enxofre, proveniente das águas contaminadas por surfactantes (detergentes), da ordem de $0,5 \mathrm{mg} . \mathrm{l}^{-1}$, valor considerado acima da concentração estabelecida pela EPA - Environmental Protection Agency - EUA (Barbosa \& Valério 2009), está contribuindo para a não biodisponibilidade de metais pesados, com a formação de sulfetos metálicos insolúveis nos sedimentos de fundo.

Os resultados da extração total de metais pesados, quando comparados com os valores-guia de qualidade de sedimentos TEL e PEL, apresentados na figura

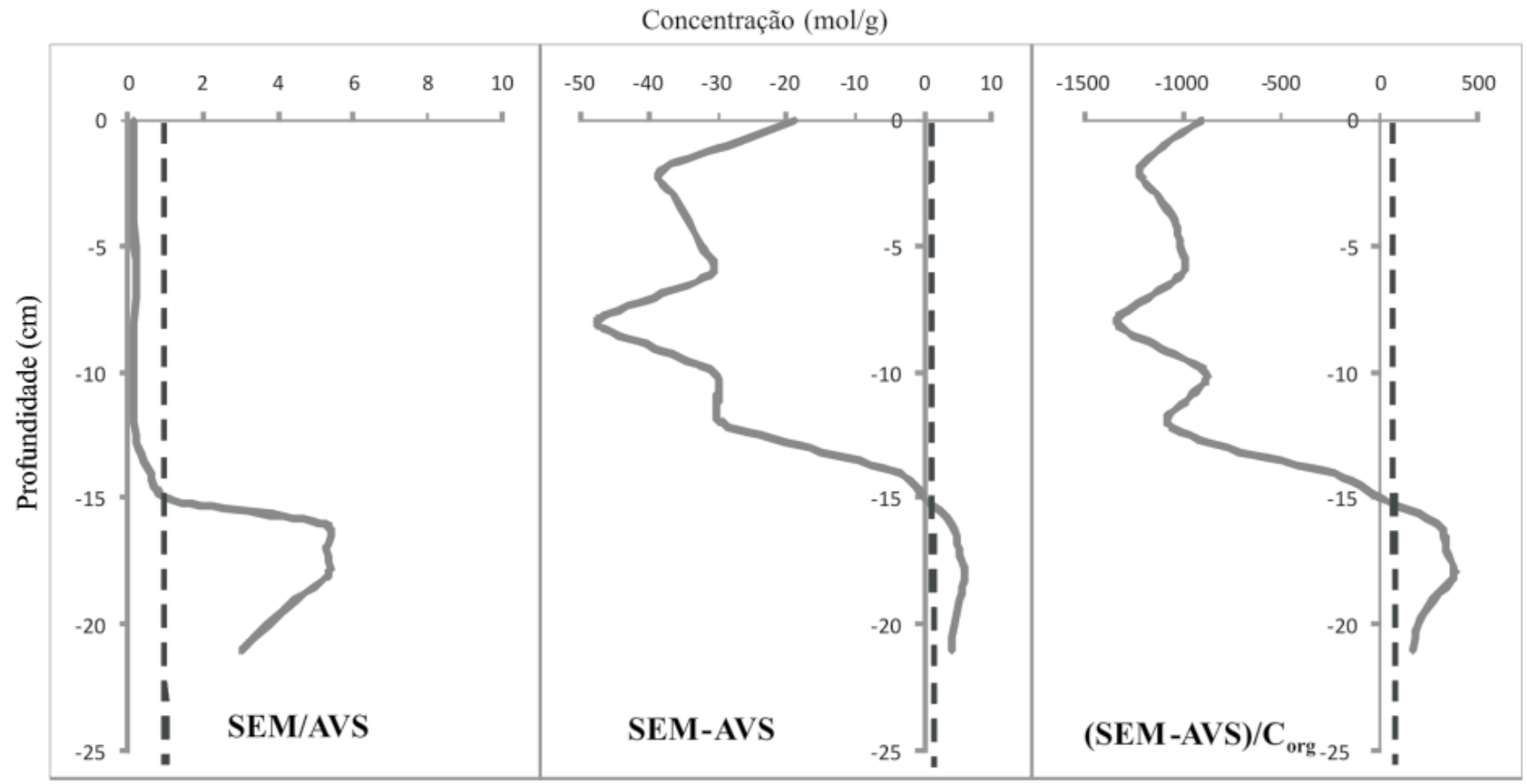

Figura 5 - Relacionamento SEM e AVS, determinados nos sedimentos de fundo, na estação de amostragem de Pirapora, em perfil de profundidade, utilizando os critérios de avaliação propostos. 
6 não retratam o observado, de acordo com os critérios de biodisponibilidade utilizados, pois além de não considerarem o fundo geoquímico natural local (background), também ignoram os processos de complexação de metais e os vários níveis de biodisponibilidade.
Desta forma, por meio das comparações entre os valores-guia PEL e TEL e às concentrações médias de metais obtidas, apresentariam, para toda a bacia do alto Tietê, efeitos de toxidez de metais nos sedimentos fluviais, o que não ocorre na realidade.
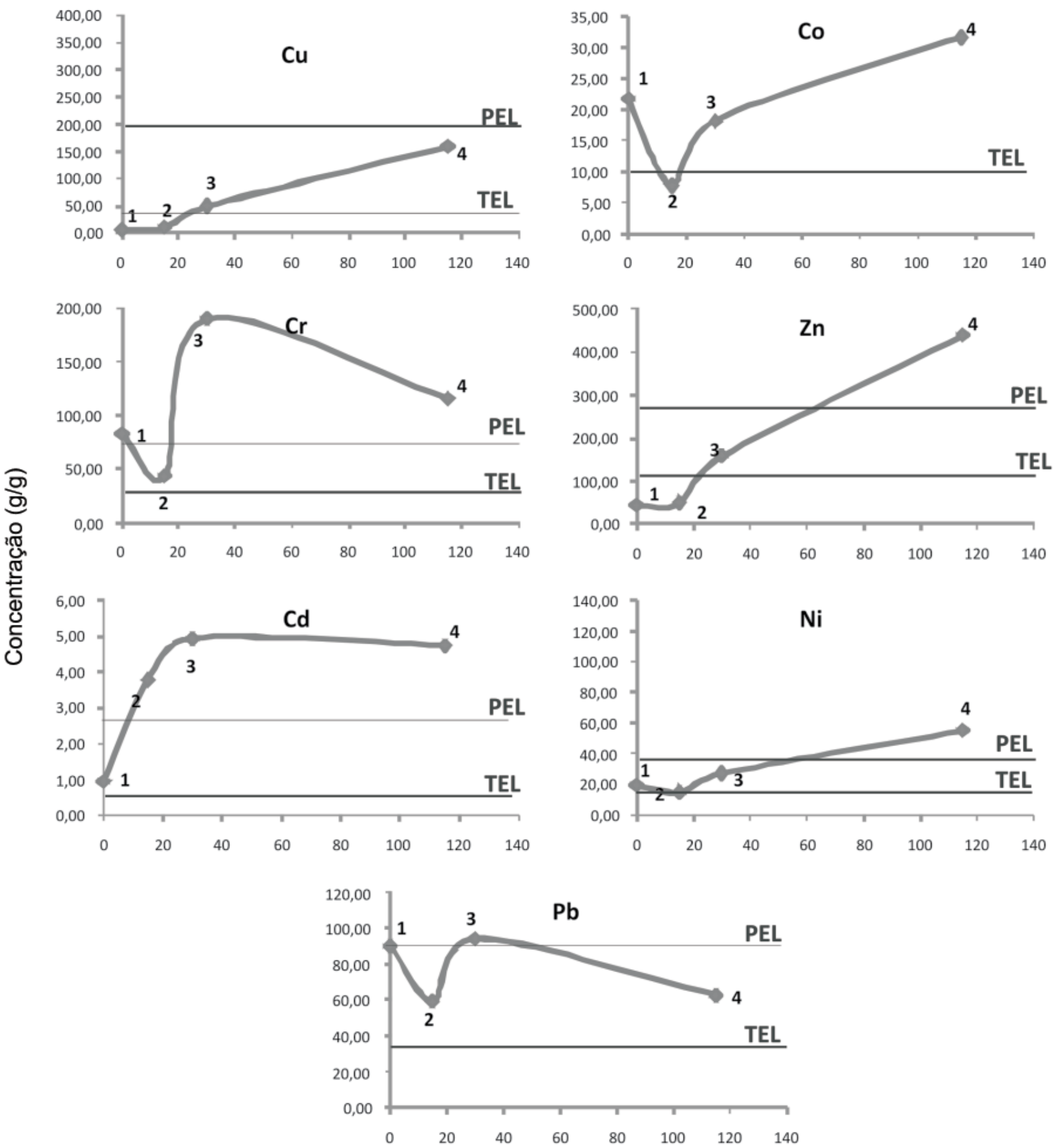

\section{Distância (km)}

Figura 6 - Variabilidade espacial das concentrações médias dos principais metais pesados extraídos, em cada perfil de sedimentos de fundo, em suas respectivas estações de amostragem: 1 - Ponte Nova, 2 - Biritiba, 3 - Mogi das Cruzes, 4 - Pirapora, em comparação com os valores-guia para sedimentos TEL e PEL. 
CONCLUSÃO Com a utilização do sistema de volatilização de sulfetos (AVS) e extração simultânea de metais pesados após ataque ácido (SEM), foi possível avaliar o potencial de biodisponibilidade de metais pesados na bacia do Alto Tietê, de acordo com os diferentes critérios utilizados. A comparação desses critérios evidenciou que, tanto SEM/AVS quanto SEM-AVS, superestimaram as condições de biodisponibilidade, principalmente quando as concentrações de AVS eram muito baixas. Ao contrário, o critério (SEM-AVS)/C se mostrou eficiente para avaliar a condição de biodisponibilidade de metais pesados, uma vez que pondera a complexação associada à matéria orgânica.
Ficou também evidenciado que a simples comparação dos valores-guia TEL e PEL, junto à extração total de metais, não satisfaz as condições para avaliar possíveis toxicidades de metais para o meio, demonstrando resultados distintos. Isto ocorre, pois, tal método não considera o fundo geoquímico natural local e também a associação dos metais com os sulfetos, superestimando essas avaliações ao longo da bacia do alto rio Tietê.

Agradecimentos Os autores agradecem à FAPESP, Fundação de Amparo à Pesquisa do Estado de São Paulo, pelo suporte financeiro (processo 2008/08774-7).

\section{Referências}

Allen H.E., Fu G., Deng B. 1993. Analysis of Acid Volatile Sulfide (AVS) and simultaneously extracted metals (SEM) for the estimation of potential toxicity in aquatic sediments. Environmental Toxicology and Chemistry, 12(8):1441-1453.

Almeida C.A. \& Rocha O. 2006. Estudo comparativo da qualidade dos sedimentos dos reservatórios do rio Tietê (SP). J. Braz. Soc. Ecotoxicol., 1(2):141-145.

Barbosa A.C. \& Valério C. 2009. Formação de espumas e gás sulfídrico $\left(\mathrm{H}_{2} \mathrm{~S}\right)$ no rio Tietê, município de Pirapora do Bom Jesus, e sua relação com o sistema de tratamento das águas do rio Pinheiros. In: IPABHi, Seminário de Rec. Hídricos da Bacia Hidrográfica do Paraíba do Sul, 2, Anais, p. 199-206.

Bevilacqua J.E., Silva I.S., Lichtig J., Masini J.C. 2009. Extração seletiva de metais pesados em sedimentos de fundo do rio Tietê, São Paulo. Química Nova, 32(1):2633.

CCME - Canadian Council of Ministers of the Environment. 1995. Protocol for the derivation of Canadian Sediment quality guidelines for the protection of aquatic life. Otawa, CCME, 35 p.

Cunha D.G.F. \& Calijuri M.C. 2008. Comparação entre os teores de matéria orgânica e as concentrações de nutrientes e metais pesados no sedimento de dois sistemas lóticos do Vale do Ribeira de Iguape, SP. Revista de Engenharia Ambiental, 5(2):24-40.

DAEE - Departamento de Águas e Energia Elétrica. 2006. Plano Estadual de Recursos Hídricos: 2004/2007. São Paulo, DAEE, Relatório Técnico, p. 92.

Di Toro D.M, Mahony J.D., Hansen D.J., Scott K.J., Hicks M.B., Mayr S.M., Redmond M.S. 1990. Toxicity of cadmium in sediments: the role of acid volatile sulfide. Environmental Toxicology and Chemistry, 9:1487-1502.

Di Toro D.M., McGrath J.M., Hansen D.J., Berry W.J., Paquin P.R., Mathew R., Wu K.B., Santore R.C. 2005. Predicting Sediment Metal Toxicity Using a Sediment Biotic Ligand Model: Methodology and Initial Application. Environ Tox. Chem., 24(10):2410-2427.

Di Toro D.M., Mahony J.D., Hansen D.J., Scott K.J., Carlson A.R., Ankley G.T. 1992. Acid volatile sulphide predicts the acute toxicity of cadmium and nickel in sediments. Environmental Science and Technology, 26:96-101.
EMBRAPA - Empresa Brasileira De Pesquisa Agropecuária. 1999. Sistema Brasileiro de Classificação de Solos. Brasília, Centro Nacional de Pesquisa de Solos, p. 142.

Hansen D.J., Berry W.J., Mahony J.D., Boothman W.S., Di Toro D.M., Robson D.L., Ankley G.T., Ma D., Yan Q., Pesch C.E. 1996. Predicting the toxicity of metal-contaminates field sediments using interstitial concentration of metals and acid-volatile sulphide normalizations. Enviromental Toxicology Chemistry, 15:2080-2094.

Lacerda L.D. \& Marins R.V. 2006. Geoquímica de sedimentos e o monitoramento de metais na plataforma continental nordeste oriental do Brasil. Geochimica Brasiliensis, 20(1):123-135.

Mortatti J., Bonassi J.A., Lopes R.A., Nolasco M.R. 2008. Determinação condutimétrica de sulfetos volatilizados por ataque ácido (AVS) e extração de metais pesados (SEM) em sedimentos de fundo ao longo do rio Tietê (SP). Geochimica Brasiliensis, 22(2):2-13.

Mozeto A.A. 2001. Critérios de Qualidade de Sedimentos (CQS) para metais pesados: Fundamentos teóricos e técnicos para implementação. São Carlos, FAPESP, Relatório Técnico-científico, 86 p.

Mozeto A.A., Nascimento M.R.L. do, Silva E.F.A, Fioravanti M.I.A. 2007. Avaliação da contaminação por metais e metalóides (água, sedimento e peixe) no rio São Francisco em Três Marias (MG - Brasil): Projeto de pesquisa participativa com a comunidade local. São Carlos, UFSCar, Relatório Técnico-científico, 68 p.

Murray K.S., Cauvet D., Lybeer M., Thomas J.C. 1999. Particle Size and Chemical Control of Heavy Metals in Bed Sediment from the Rouge River, Southeast Michigan. Environmental Science \& Techmo, 33:997992.

Oliveira J.D., Fava F.H., Valente J.P.S., Florentino A.O., Padilha P.M. 2006. Estudo da biodisponibilidade de $\mathrm{Pb}, \mathrm{Cu}, \mathrm{Cr}, \mathrm{Zn}, \mathrm{Mn}$ e Ni em amostras de sedimentos do Ribeirão Lavapés, Botucatu-SP. In: SBQ, Reunião Anual da Sociedade Brasileira de Química, 29, Resumos, 70 p.

Samuel J., Rouault R., Besnus Y. 1985. Analyse multiémentaire standardisée des matériaux géologiques en spectrométrie d'émission par plasma a couplage inductif. Analusis, 13:312-317. 
Silva I.S., Abade G., Lichtig J., Masini J.C. 2002. Heavy metal distribution in recent sediments of the Tietê Pinheiros river system in São Paulo state, Brazil. Applied Geochemistry, 17:105-116.

Silvério P.F. 2003. Bases técnico-cientificas para a derivação de valores-guia de qualidade de sedimentos para metais: Experimentos de campo e laboratório. Tese de Doutorado, Escola de Engenharia de São Carlos, Universidade de São Paulo, São Carlos, 145 p.

Silvério P.F., Nascimento M.R.L., Mozeto A.A. 2006. Valores-guia de qualidade de sedimentos de ambientes aquáticos continentais e valores de referência de metais e metalóides em sedimentos. In: Mozeto, A.A., Umbuzeiro G.A., Jardim W.F. (eds.) Métodos de coleta, análises físico-químicas e ensaios biológicos e ecotoxicológicos de sedimentos de água doce. São Carlos, Cubo Multimídia, p. 71-89.

Smith L.S., Macdonald D.D., Keenleyside K.A., Ingersoll C.G., Field L.J. 1996. A Preliminary Evaluation of Sediment Quality Assessment Values for Freshwater Ecosystems. Journal Great Lakes Res., 22(3):624-638.

USEPA - U.S. Environmental Protection Agency, 2000. Methods for measuring the toxicity and bioaccumulation of sediment - associated contaminants with freshwater invertebrates - Second Edition - EPA/600/R-99/064. Duluth, Guidance Manual.

Manuscrito ID 18237

Submetido em 09 de agosto de 2010

Aceito em 13 de outubro de 2011 